PREPARED FOR THE U.S. DEPARTMENT OF ENERGY, UNDER CONTRACT DE-AC02-76CH03073

PPPL-3616

PPPL-3616

UC-70

Observation of Spontaneous Neoclassical Tearing Modes

by

E.D. Fredrickson

October 2001

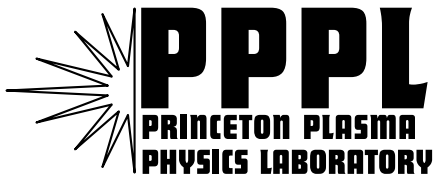

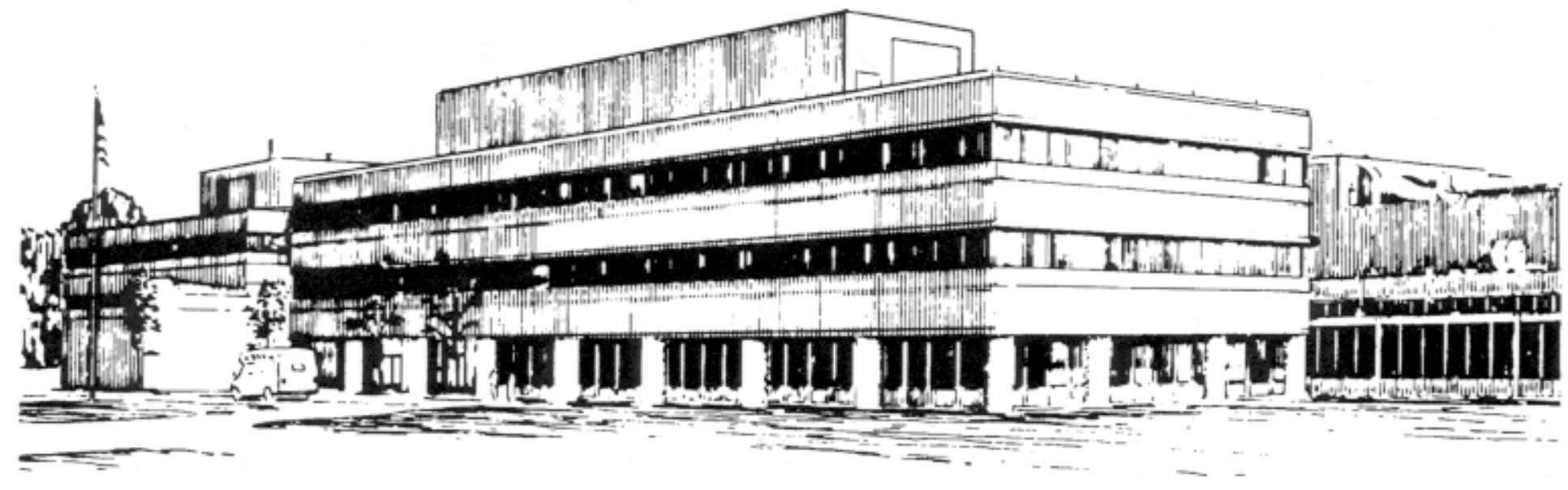

PRINCETON PLASMA PHYSICS LABORATORY PRINCETON UNIVERSITY, PRINCETON, NEW JERSEY 


\section{PPPL Reports Disclaimer}

This report was prepared as an account of work sponsored by an agency of the United States Government. Neither the United States Government nor any agency thereof, nor any of their employees, makes any warranty, express or implied, or assumes any legal liability or responsibility for the accuracy, completeness, or usefulness of any information, apparatus, product, or process disclosed, or represents that its use would not infringe privately owned rights. Reference herein to any specific commercial product, process, or service by trade name, trademark, manufacturer, or otherwise, does not necessarily constitute or imply its endorsement, recommendation, or favoring by the United States Government or any agency thereof. The views and opinions of authors expressed herein do not necessarily state or reflect those of the United States Government or any agency thereof.

\section{Availability}

This report is posted on the U.S. Department of Energy's Princeton Plasma Physics Laboratory Publications and Reports web site in Fiscal Year 2002. The home page for PPPL Reports and Publications is: http://www.pppl.gov/pub_report/

DOE and DOE Contractors can obtain copies of this report from:

U.S. Department of Energy

Office of Scientific and Technical Information

DOE Technical Information Services (DTIS)

P.O. Box 62

Oak Ridge, TN 37831

Telephone: (865) 576-8401

Fax: (865) 576-5728

Email: reports@adonis.osti.gov

This report is available to the general public from:

National Technical Information Service

U.S. Department of Commerce

5285 Port Royal Road

Springfield, VA 22161

Telephone: 1-800-553-6847 or

(703) 605-6000

Fax: (703) 321-8547

Internet: http://www.ntis.gov/ordering.htm 


\title{
Observation of Spontaneous Neoclassical Tearing Modes
}

\author{
E. D. Fredrickson \\ Princeton Plasma Physics Laboratory \\ Princeton, New Jersey 08543
}

\begin{abstract}
We present data in this paper from the Tokamak Fusion Test Reactor (TFTR) which challenges the commonly held belief that extrinsic MHD events such as sawteeth or ELMs are required to provide the seed islands that trigger Neoclassical Tearing Modes (NTMs). While sawteeth are reported to provide the trigger for most of the NTMs on DIII-D and ASDEX-U, the majority of NTMs seen in TFTR occur in plasmas without sawteeth, that is which are above the $\beta$ threshold for sawtooth stabilization. Examples of NTMs appearing in the absence of any detectable extrinsic MHD activity will be shown. Conversely, large $n=1$ modes in plasmas above the NTM $\beta$ threshold generally do not trigger NTMs. An alternative mechanism for generating seed islands will be discussed.
\end{abstract}




\section{Introduction}

Tearing modes were discovered on the first tokamak, T3 [1] and have been seen on every tokamak since. Their deleterious affect on confinement and potential to lead to disruptions has been well documented. A theoretical model, hereinafter referred to as the "classical" model, has been developed which describes the stability and evolution of islands in low beta plasmas [2-4]. This model generally predicts the $2 / 1$ mode to be marginally stable and higher $\mathrm{n}$ modes to be stable, in reasonable agreement with observed tearing mode activity in early tokamak experiments.

When high beta, low collisionality plasmas were first created on the Tokamak Fusion Test Reactor, TFTR [5], tearing modes were seen under conditions where the classical theoretical model predicted stability. In particular the $3 / 2$ and $4 / 3$ tearing modes were seen, in addition to the $2 / 1$ mode. It is very difficult to find current profiles which are unstable to these modes and for which the predicted saturated island size is significantly greater than some fraction of a percent of the minor radius. These plasmas were different from plasmas in earlier machines in that the collisionality was low enough and the poloidal beta high enough so that there was substantial bootstrap current.
It was subsequently shown that the growth and evolution of the island width could reasonably well be fit with "neoclassical" tearing mode (NTM) theory [6,7]. In this model the density and temperature gradients which drive, through neoclassical effects, the bootstrap current, are assumed to be flattened across the island 'O-point'. This flattening reduces the local bootstrap current drive, introducing a helical perturbation in the bootstrap current, which in turn causes the island to grow larger. This extension of the classical (low $\beta$ ) tearing mode model to high $\beta$, collisionless plasmas has serious implications for fusion reactors. Not only are more modes potentially unstable, but the modes will be driven to larger amplitudes by the inclusion of neoclassical drive terms.

The simple implementation of this model had a serious flaw in that this neoclassical drive was sufficiently strong so that many of the low order tearing modes were predicted to be unstable. This contradicts the experimental observation that typically only one or two modes were observed at one time, and in many similar plasmas no modes were seen. It was soon realized that a partial resolution of this paradox lay in the observation that small islands would not be able to effectively flatten the pressure gradient $[8,9]$. Near the island separatrix the poloidal connection length 
becomes long and the large parallel conductivity can no longer dominate the weaker perpendicular diffusivity. Thus, for small enough islands, the pressure profile is not flattened and the bootstrap current is not perturbed. This creates a situation where a "seed" island is necessary to initially flatten the pressure gradient and trigger the now metastable modes. While this resolved the paradox, it made the task of predicting the appearance of specific islands more difficult.

As research, theoretical and experimental, continued in this area, additional physical insights were gained. In particular, it was realized that the propagation of small islands through the plasma would induce resonant current perturbations affecting mode stability $[10,11]$. Depending on the direction of propagation of the initial, small, island, the current induced by the ion polarization drift could be stabilizing or destabilizing. It is generally assumed to be stabilizing, but apart from the parametric scaling, the magnitude of this term, like other terms in neoclassical theory, is only predicted approximately.

In previous works, the utility of the neoclassical tearing mode theory in describing many features of tearing modes in present tokamaks has been well documented[12-14]. In this paper we will investigate aspects of the experimental observations of tearing modes that do not fit so well with the theory, or where the theory is clearly incomplete.

Clear sources of the seed islands were not identified for most neoclassical tearing modes on TFTR. Sawteeth are generally absent in plasmas with NTMs and of course TFTR does not usually have ELMs. The occasionally observed onset of $3 / 2$ NTMs near the time of a sawtooth crash supports the model that sawtooth induced seed islands can trigger NTMs. However, a large $n=1$ ideal mode, related to the fishbone instability [15], is often present in plasmas with $\beta$ well above the NTM threshold, but does not generally trigger NTMs, suggesting that the model of non-linear coupling of the 1/1 mode to higher n's is incomplete.

The model that non-linear coupling of the $\mathrm{n}=1$ mode to $\mathrm{n}=2$ and higher $\mathrm{n}$ 's is further challenged in that it would be expected that the coupling of the $1 / 1$ to the $2 / 1$ or $3 / 2$ would be stronger than the coupling to higher n's. Yet in many regimes it is the $5 / 4$ or $4 / 3$ modes which are more common than the $2 / 1$ or $3 / 2$.

Minor $\beta$-limit disruptions, even with weak precursors, nearly always trigger neoclassical tearing modes, whereas the larger fishbonelike mode seldom if ever triggers NTMs. This suggests that it is not the $1 / 1$ mode, per se, that provides the trigger, but perhaps a subsidiary effect associated with the magnetic 
reconnection event. Certainly, the observation of very fast heat pulses following sawtooth crashes have suggested that the effects of the reconnection extend well beyond the reconnection radius.

As the high performance, "supershot", regime was extended to higher currents (the original supershot plasmas were at plasma currents of $0.7-0.9 \mathrm{MA}$ ), the character of the neoclassical activity changed dramatically. Rather than the $3 / 2$ and 2/1 modes, which were so deleterious to performance at low plasma current, at intermediate currents of $1.4-2.0$ MA the dominant mode activity became $3 / 2$ and $4 / 3$ modes. At the highest currents [2.0 $2.5 \mathrm{MA}, \mathrm{q}(\mathrm{a}) \approx 4]$ the dominant mode activity became $4 / 3$ and $5 / 4$ modes.

With the higher mode numbers, the effect on performance became weaker until with $5 / 4$ modes the effects were largely negligible. The presence of the 5/4 modes could actually be beneficial in that the NTM near the core limited the pressure profile peaking responsible for beta limiting disruptions, without significantly degrading global confinement.

We will begin, in the next section, with a description of the TFTR tokamak, the relevant operating regimes, and the most relevant diagnostics used for this study. In Sect. III we describe the theoretical framework in which the experimental observations are analyzed. In Sect. IV we present the experimental data, with a minimum of discussion. In Sect. V we discuss the implications of the observations reported in Sect. IV and finally in Sect. VI we summarize our conclusions.

\section{Experimental method and diagnostics}

The data discussed in this paper are generally from enhanced confinement (beam heated supershot) discharges in the TFTR, the only TFTR regime where NTM activity was common.

The TFTR is a circular cross section machine, which routinely operated with toroidal field up to $5.9 \mathrm{~T}$, plasma current up to 2.6 MA, density typically 0.3 to 0.6 Greenwald $\left(3-7 \times 10^{19} / \mathrm{m}^{3}\right)$ and electron temperatures from 4 to $10 \mathrm{keV}$. Standard plasma major radii were $2.45 \mathrm{~m}, 2.52 \mathrm{~m}$ and $2.62 \mathrm{~m}$ with corresponding minor radii of 0.8 $\mathrm{m}, 0.87 \mathrm{~m}$ and $0.97 \mathrm{~m}$ respectively. The plasmas discussed in this paper were heated with up to $40 \mathrm{MW}$ of neutral beam injection (NBI) power at beam energies of up to 120 $\mathrm{keV}$.

The plasmas were typically limited on a toroidal, inboard carbon limiter. Recycling of hydrogenic species and carbon from the limiter determined the minimum plasma density and the best confinement and highest stored 
energies were achieved when this recycling was minimized (i.e., the supershot regime).

The principle diagnostics for study of the tearing modes are the Mirnov coil arrays, which measure magnetic fluctuations at the plasma edge, and the two microwave Grating Polychromators (GPC) which measure the second harmonic electron cyclotron emission (ECE). The fast electron temperature profile measurements and the full toroidal and poloidal Mirnov array data is typically only available in one to four discrete time windows, each about $20 \mathrm{~ms}$ long.

The Mirnov coil arrays provide a measure of the poloidal and toroidal structure of the modes, the toroidal mode number, $\mathrm{n}$ and the poloidal mode number, $\mathrm{m}$. The arrays also provide the absolute magnitude of the magnetic fluctuations at the vacuum vessel wall, which can be used to benchmark a linear eigenfunction calculation and derive an island size.

The data are analyzed with Fourier transforms to find the amplitude and relative phases of signals from each of the coils. The best fit to the phase data is then used to identify the mode numbers. The poloidal structure of the amplitude is then calculated, from which an effective poloidal field fluctuation amplitude is then determined.
The TFTR vacuum vessel and plasma cross-sections are circular and typically the plasma is not centered in the vacuum vessel. Thus, the ratio of the minor radius of the Mirnov coils to the plasma minor radius varies from $\approx 1.1$ on the inboard side to $\approx 1.6$ on the outboard side with the poloidal average being about 1.4 (for an $80 \mathrm{~cm}$ plasma). Empirically it has been found that using the poloidally averaged amplitude and an effective radius of the Mirnov coil array approximately the radius of the vacuum vessel (e.g. for $\mathrm{a}=80 \mathrm{~cm}$ cases $\mathrm{r}_{\text {wall }} / \mathrm{a} \approx 1.4$ ) gives reasonable agreement with the island sizes measured with the GPC.

The time evolution and onset amplitude are inferred indirectly using a single Mirnov coil signal digitized at a $100 \mathrm{kHz}$ rate whose amplitude is normalized to the full poloidal data at one or more times. While this gives a qualitatively correct estimate for the mode amplitude evolution, as the plasma equilibrium evolves (e.g., the Shafranov shift changes), the scale factor will change slightly.

The GPC's provide a 20 point internal measurement of the electron temperature profile. The measurements are made at discrete frequencies, which are then mapped to spatial locations by assuming they originate at locations of the $2^{\text {nd }}$ harmonic of the electron cyclotron frequency. Mapping can be done reasonably accurately using just the vacuum 
toroidal field, but a more accurate map can also be done using the $\bmod (B)$ as calculated with an equilibrium code.

Utilizing Fourier analysis it is possible to reduce the effective noise level of the system to of order $0.2 \%$. Translating this sensitivity into an equivalent island size is a complex process. Nevertheless, for larger islands it is possible to constrain the reasonable range of island sizes as well as to identify the radial locations of the mode rational surfaces - an important constraint in calculating the equilibrium necessary for the island evolution simulations.

\section{Analysis approach: Modified}

\section{Rutherford equation}

The Mirnov data will be interpreted by comparison of experimental measurements to the predictions of a quasi-cylindrical, timedependent $\Delta^{\prime}$ stability code. The magnetic fluctuation amplitude as measured at the vacuum vessel wall can be related to the island width through the shape of the linear eigenfunction. Adjustable parameters in the neoclassical terms are used to fit the saturated island width, the growth rate and the threshold island size. The code uses the equilibrium and bootstrap current profile evolution as calculated with the TRANSP code[16].
Tearing mode stability is calculated from a fourth order partial differential equation. The solution can be reduced to a boundary layer formalism where the stability is governed by the solution to the second order partial differential equation in the "external" region [2]. The stability is expressed in terms of the discontinuity of the derivative across the boundary (tearing) layer. For islands with width greater than the tearing layer the growth rate is proportional to this normalized discontinuity, the $\Delta^{\prime}$, as described by the Rutherford Equation [3].

This equation is valid in a regime where the island growth is linear in time and slower than the resistive flux diffusion time across the tearing layer. The eigenvalue equations are solved with the "constant- $\psi$ " approximation.

Neoclassical and other modifications to the classical tearing mode theory are generally included in this Rutherford equation. The island evolution is then described by the modified Rutherford equation

$$
\begin{aligned}
& \tau_{\mathrm{R}} \partial \mathrm{w} / \partial \mathrm{t}= \\
& \quad \Delta^{\prime}(\mathrm{w})+\mathrm{k}_{\mathrm{nc}} \Delta_{\mathrm{nc}}-\mathrm{k}_{\mathrm{pol}} \Delta_{\mathrm{pol}}-\Delta_{\mathrm{GGJ}}
\end{aligned}
$$

In this equation $1 / \tau_{\mathrm{R}}=1.22 \mathrm{a}^{2} \eta / \mu$ where $\eta$ is the plasma resistivity and $\mu$ is the magnetic permeability. The island width, $w$ and the $\Delta^{\prime}(\mathrm{w})$ are normalized to the plasma minor 
radius. The neoclassical term reflects the drive resulting from the "missing" bootstrap current. The polarization term has been mentioned previously and the last term is the GlasserGreene-Johnson (GGJ) term [17] reflecting the stabilizing effect of the average good curvature of tokamaks.

The quasi-cylindrical, zero beta $\Delta^{\prime}$ is calculated by numerically solving the external equation governing the perturbed helical flux function

$$
\begin{aligned}
& {\left[\partial^{2} / \partial r^{2}+1 / r \partial / \partial r-m^{2} / r^{2}-\right.} \\
& \left.\left(\partial J_{0} / \partial r\right) /\left(\partial \psi_{0} / \partial r\right)\right] \psi_{m, n}=0 .
\end{aligned}
$$

The $J(r)$ is calculated in TRANSP from the resistive current diffusion equation. The q(r) is calculated by the VMEC equilibrium code and is used to calculate $\partial \psi_{0} / \partial$ r. Equation 2 is integrated outward from the axis to $r_{s}-\delta r^{(-)}$ and from the wall inward to $r_{s}+\delta r^{(+)}$. The $\psi_{\mathrm{m}, \mathrm{n}}$ is matched across this layer and the normalized discontinuity in the derivative of $\psi_{\mathrm{m}, \mathrm{n}}$ is $\Delta^{\prime}(0)$. The $\Delta^{\prime}(\mathrm{w})$ is found by using $\psi_{0}$ to identify the inner and outer locations of the island edges and then $\psi_{m, n}$ is matched across the island by making the "constant- $\psi$ " approximation.

In plasmas with large pressure driven currents (bootstrap current), the local flattening of the pressure due to the island reduces the local bootstrap current, which increases the island size. A heuristic derivation [18] results in an expression of the form

$$
\Delta_{\mathrm{nc}}=16 \mathrm{~J}_{\mathrm{bs}} /(\mathrm{s} \mathrm{w}<\mathrm{J}>)
$$

where $\mathrm{w}$ is the island width, $\mathrm{s}$ ( $\left.\equiv \mathrm{r} \mathrm{q}^{\prime} / \mathrm{q}\right)$ is the magnetic shear at the radius of the mode rational surface, $r_{s}$, and $B_{\theta}$ is written in terms of $\langle\mathrm{J}\rangle$, the average current density for the region $0<\mathrm{r}<\mathrm{r}_{\mathrm{s}}$. The derivation is not exact and a coefficient, $\mathrm{k}_{\mathrm{nc}}$, has been introduced into Eq. 1.

The calculation of the bootstrap current is fairly complicated and previous studies of neoclassical tearing modes have used an approximate formulation for the local bootstrap current

$$
\mathrm{J}_{\mathrm{bs}} \propto 0.25 \varepsilon^{1 / 2} \beta_{\mathrm{pol}}<\mathrm{J}>/ \mathrm{L}_{\mathrm{p}}
$$

where $\varepsilon=r_{s} / R$ is the inverse aspect ratio, and $\mathrm{L}_{\mathrm{P}}$ is the pressure gradient scale length. Using this expression in Eq. 3 gives

$$
\Delta_{\mathrm{nc}} \approx 4 \varepsilon^{1 / 2} \beta_{\mathrm{pol}} \mathrm{r}_{\mathrm{s}} / \mathrm{s} \mathrm{w} \mathrm{L}_{\mathrm{p}}
$$

The choice of the factor of 4 is somewhat arbitrary, but consistent with the factor of 16 
heuristically derived in Eq. 3. Various theoretical treatments have estimated this constant to be as low as 3.17 [19] and as high as 9.26 [20]. The actual best fit of this parameter to experimental data has varied from as low as $1.7[12,21]$ to 7 [14].

This simple model for $\Delta_{\mathrm{nc}}$ will not scale exactly with the evolution of the bootstrap current as the evolution of the electron and ion density and temperature gradients do not necessarily evolve in the same manner. For that reason, to improve the reliability of the scaling between the different regimes studied in the following sections, the more correct expression for the bootstrap term will be used and the TRANSP calculation of the bootstrap current evolution will be used.

The effect of the island on the local $\mathrm{P}^{\prime}$, and thus $\mathrm{J}_{\mathrm{BS}}$, is also difficult to calculate, but depends on, among other parameters, the ratio of the parallel to perpendicular thermal conductivity. An expression has been derived to approximate this effect by multiplying the above expressions for the bootstrap term by $\mathrm{w}^{2}$ / $\left(\mathrm{w}^{2}+\mathrm{k}_{\mathrm{d}} \mathrm{w}_{\mathrm{d}}^{2}\right)$ [8]. Again, a fudge factor, $\mathrm{k}_{\mathrm{d}}$ has been introduced to allow for fitting to experimental data. The critical island width, $\mathrm{w}_{\mathrm{d}}$, is estimated to be

$$
\mathrm{w}_{\mathrm{d}} \approx 5.1 \mathrm{r}_{\mathrm{s}}\left(\chi_{\perp} / \chi_{\|}\right)^{1 / 4} /(\varepsilon \mathrm{s} \mathrm{n})^{1 / 2},
$$

where $\mathrm{n}$ is the toroidal mode number and $\chi_{\perp}$ and $\chi_{\|}$are the perpendicular and parallel thermal diffusivities, respectively. The effective $\chi_{\|}$should be the lesser of the collisional and collisionless expressions [14].

The last two terms are the "GlasserGreene-Johnson" and the "polarization drift" terms which are stabilizing. An approximate form for the Glasser-Greene-Johnson term is given in Ref. 19, Eq. 51:

$$
\Delta_{\mathrm{GGJ}}=-5.4\left(\beta_{\mathrm{p}} \varepsilon^{2} \mathrm{~L}_{\mathrm{q}}{ }^{2}\right) /\left(\mathrm{rw} \mathrm{L}_{\mathrm{P}}\right)\left(1-\mathrm{q}^{2}\right) / \mathrm{q}^{2} .
$$

The $\Delta_{\mathrm{GGJ}}$ term results from the average good curvature in a tokamak plasma and is mentioned here for completeness. It is generally small in TFTR and will be neglected in the remainder of this paper.

The simplified form for the polarization drift term commonly used is:

$$
\Delta_{\mathrm{pol}} \propto-\mathrm{g}\left(\rho^{2}{ }_{\theta i} \beta_{\mathrm{p}} \mathrm{g}\right) / \mathrm{w}^{3}\left(\mathrm{~L}_{\mathrm{q}} / \mathrm{L}_{\mathrm{P}}\right)^{2} .
$$

The parameters $L_{q}$ and $L_{P}$ are the $q$ and pressure gradient scale lengths, respectively. The ion poloidal gyroradius is $\rho_{\theta i}$, the ion collision frequency is $v_{\mathrm{i}}$ and $\varepsilon$ is the local aspect ratio. The parameter $\mathrm{g}$ approaches unity for $v_{\mathrm{i}} / \omega_{*_{\mathrm{e}}}<<1$ and $\varepsilon^{3 / 2}$ in the other 
extreme. Theoretical estimates for the multiplier on this term are also uncertain, but in the range of $10-20$.

Both of these terms become large and negative for small island size, but rapidly become small as the island size increases. Thus, introducing either of these terms into the island evolution equation will introduce a condition on threshold island width for instability even when $\Delta^{\prime}(0)>0$, but their effect on the saturated island size is generally small.

The model described above has been incorporated into a code which integrates the modified Rutherford equation in time to simulate island evolution. The time dependent $\Delta^{\prime}(\mathrm{w})$ is calculated numerically on the TRANSP time base and the other terms are evaluated as well. As mentioned above, in the neoclassical tearing mode theory, there are several adjustable constants which may be used to fit the observed island evolution. The range of some of these parameters used in previous papers is quite large and the first goal in the next section will be to determine the range to which these parameters can be constrained by experimental observations. The threshold island size predicted by the model under various assumptions will also be compared to the minimum measured island size.

\section{NTM's, onset and evolution}

Neoclassical tearing modes were most often not triggered by sawteeth on TFTR and conversely large $n=1$ kink modes with $\beta$ well above the NTM $\beta$ threshold seldom if ever triggered NTMs. The lack of sawteeth to trigger the NTMs in TFTR is a consequence of the strong correlation of the $\beta$ thresholds for stabilization of sawteeth with that for NTM onset. The correlation of the sawtooth stabilization threshold with the NTM onset threshold could be coincidental, but it strongly suggests that there is underlying physics coupling the sawtooth stabilization mechanism and NTM triggers on TFTR.

The NTMs were most common in TFTR in the $\beta$ range just above the sawtooth stabilization threshold. As the plasma $\beta$ was raised in TFTR from shot to shot, the sawtooth period would become longer and the crashes larger until the sawtooth period became longer than the beam injection period. At slightly higher $\beta$ the NTMs would occur in roughly 30$50 \%$ of the discharges. Near the threshold, sawtooth crashes would occasionally be coincident with NTM onset.

For the remaining $50-70 \%$ or so of plasmas above the sawtooth stabilization threshold and without NTMs the $\beta$ would continue to increase, with only an $\mathrm{n}=1$ bursting or continuous kink-like mode present. Thus, 
NTMs on TFTR tended to onset in a relatively quiescent plasma and the lack of correlation between NTM onset and sawtooth crashes was clear.

(a)
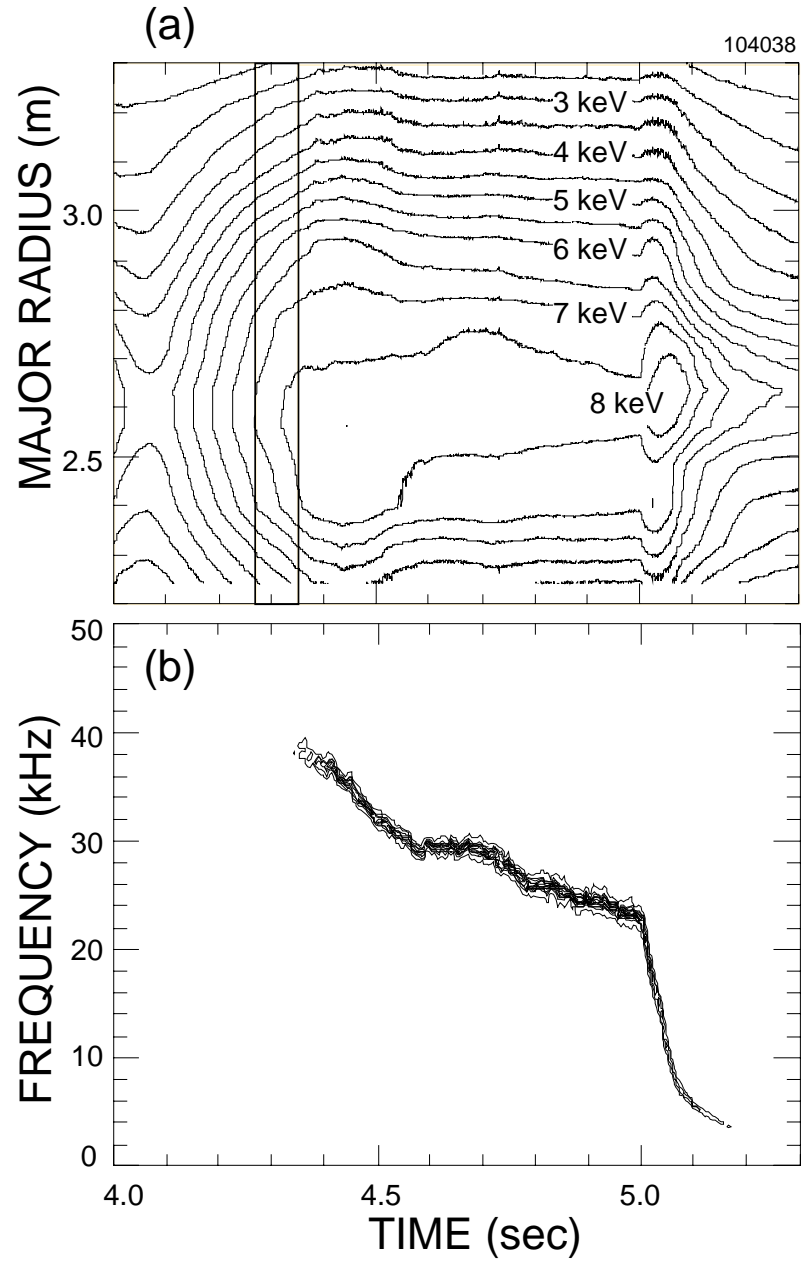

Fig. 1 (a) Contours of electron temperature for shot 104038, (b) spectrogram of Mirnov coil data for shot 104038 showing onset of 4/3 NTM in quiet plasma.

An important corollary to this observation is that in the many discharges just below the $\beta$ threshold for sawtooth stabilization, the large sawtooth crashes did not trigger NTMs; this implies that the NTMs are appearing not far above the threshold or that sawteeth did not provide effective triggers for the NTMs. The
NTM theory described here also does not explain or predict some other common, but not always present, phenomena. These phenomena include the evolution of $4 / 3$ or $5 / 4$ NTMs in the presence of a $3 / 2$ mode, the "chirping" behavior of NTMs, the evolution of an NTM through a sawtooth crash, and the initial very slow growth or quasi-saturated mode amplitude at onset.

An example of a "spontaneous" 4/3 NTM onset is shown in Fig. 1. In Fig. 1a are shown contours of the electron temperature where it can be seen that there is no indication of a reconnection event at the time of the mode onset. In Fig. $1 \mathrm{~b}$ is shown the spectrogram of fluctuations with the $4 / 3$ mode indicated. Apart from some very low level magnetic turbulence at frequencies below $5 \mathrm{kHz}$, this discharge was very quiet. Clearly sawteeth, ELMs or fishbones are not necessary to trigger NTMs. In this section we will present further data to support this claim, and suggest an alternative model for the onset of NTMs.

As has been previously reported [12,21], the simple NTM model can, however, reasonably well model, in most cases, the gross behavior of the NTMs on TFTR. For islands more than twice the threshold island size, the resistive time constant and the bootstrap drive (the $\mathrm{k}_{\mathrm{nc}}$ and $\mathrm{k}_{\mathrm{res}}$ constants) are used to match the growth and decay rates and 
the saturated island width. By comparison of simulations with measurements of the initial island width behavior, we can further place some constraints on the $\mathrm{k}_{\mathrm{d}}$ and $\mathrm{k}_{\mathrm{pol}}$ terms which determine the threshold island sizes.

We begin our study with examples of NTMs from a sequence of approximately 50 discharges where the $\beta$ was kept just above the sawtooth stabilization threshold $\left(\beta_{\mathrm{pol}} \approx 1\right)$ and the plasma external parameters were held relatively constant. This data set has been previously discussed by Chang et al. [12,21]. The toroidal field was $4.8 \mathrm{~T}$, neutral beam heating power was $\approx 16 \mathrm{MW}, \mathrm{q}(\mathrm{a}) \approx 5$, and the plasma current was $\approx 1.6 \mathrm{MA}$ with small $(100$ - $200 \mathrm{kA}$ ) current ramps up or down at the start of NBI.
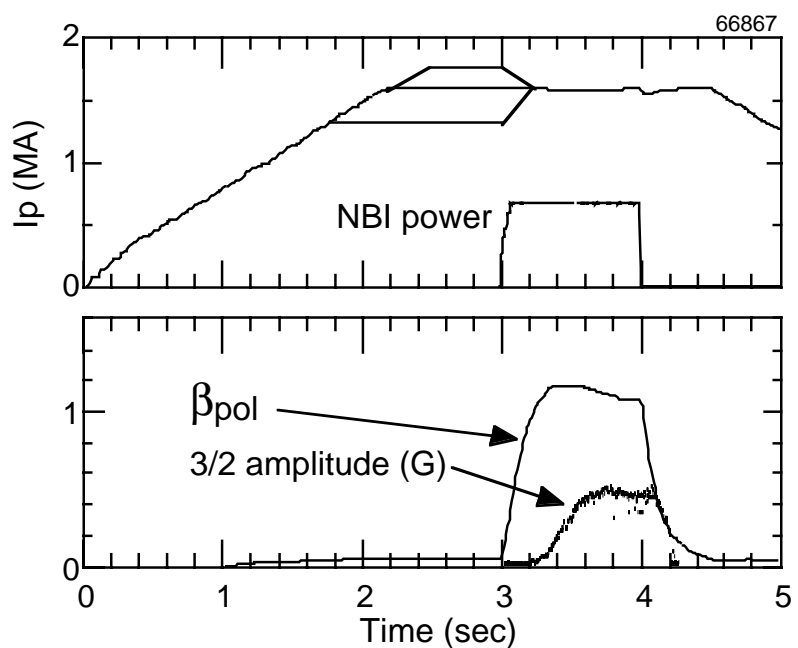

Fig. 2 Plasma current, NBI power and $\beta_{\text {pol }}$ for a typical supershot with a $3 / 2$ NTM.

In Fig. 2 are traces showing the evolution of the plasma current, the NBI heating power and the poloidal beta and 3/2 mode amplitude.
Of the $\approx 50$ plasma discharges in this sequence, 7 developed $3 / 2$ NTMs as the dominant MHD activity, 11 developed 4/3 NTMs and the remaining shots had fishbonelike modes. Additionally, many of the discharges also had 5/4 NTMs present during fishbone activity or with other NTMs.

Sawteeth were stabilized during the NBI phase for most shots, as was discussed above. NTMs typically onset 200 to $300 \mathrm{~ms}$ after the start of NBI. Only two of seven $3 / 2$ NTMs were triggered by sawteeth and none of the dominant 4/3 NTMs were triggered by sawteeth. Approximately one half of the $4 / 3$ NTMs were spontaneous with no detectable extraneous MHD events. The remainder of the 4/3 and 3/2 NTMs had an onset roughly correlated with a weak off-axis reconnection event, i.e., a partial sawtooth.

We begin fitting the mode evolution using data from a "spontaneous" 4/3 NTM. Fishbone modes are present at the time of onset, but it is not believed that they played a role in triggering this NTM. The early growth of the NTM has two stages, a slowly growing phase at low amplitude, followed by the "linear" growth expected for NTMs well above the threshold island size.

In Fig. 3 are shown contours of the electron temperature vs. major radius and in Fig. 4 is a spectrogram of the magnetic 
fluctuations. The mode onset time can be seen from Fig. 4 to be uncertain, but the onset occurs somewhere between 3.35 and $3.4 \mathrm{~s}$. There is no sawtooth crash in this time interval (indicated by the vertical lines in Fig. 3), but there is a weak partial sawtooth crash.

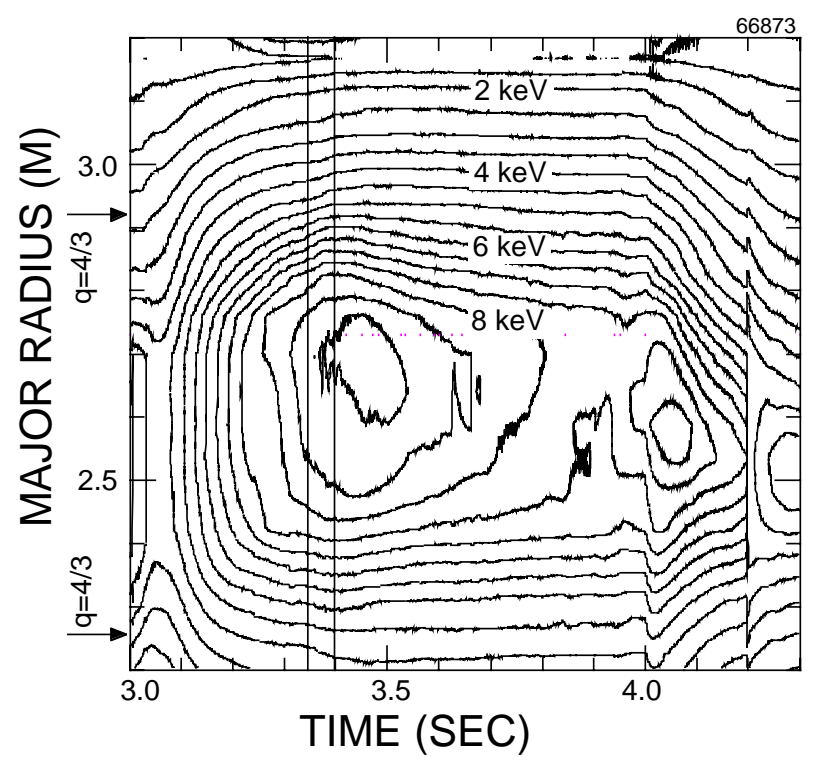

Fig. 3 Contours of electron temperature through NBI heating period. Dashed line indicates onset time of $4 / 3$ NTM at which there is no sawtooth crash.

An interesting general feature of NTMs on TFTR is also visible in Fig. 4, the frequency chirps. Very little is understood about the nature or origin of the chirps, but virtually every NTM on TFTR shows this behavior. Following each chirp, there is a measurable reduction in the neutron rate, although the drop is quite small; the largest are of order $1 \%$. This effect on the neutron rate suggests that the chirps are associated with some spatial redistribution or loss of beam fast ions, as the effect on local plasma parameters is quite small (in contrast to observations on ASDEX where the chirps were associated with relatively larger effects on $\mathrm{T}_{\mathrm{e}}$ [22].

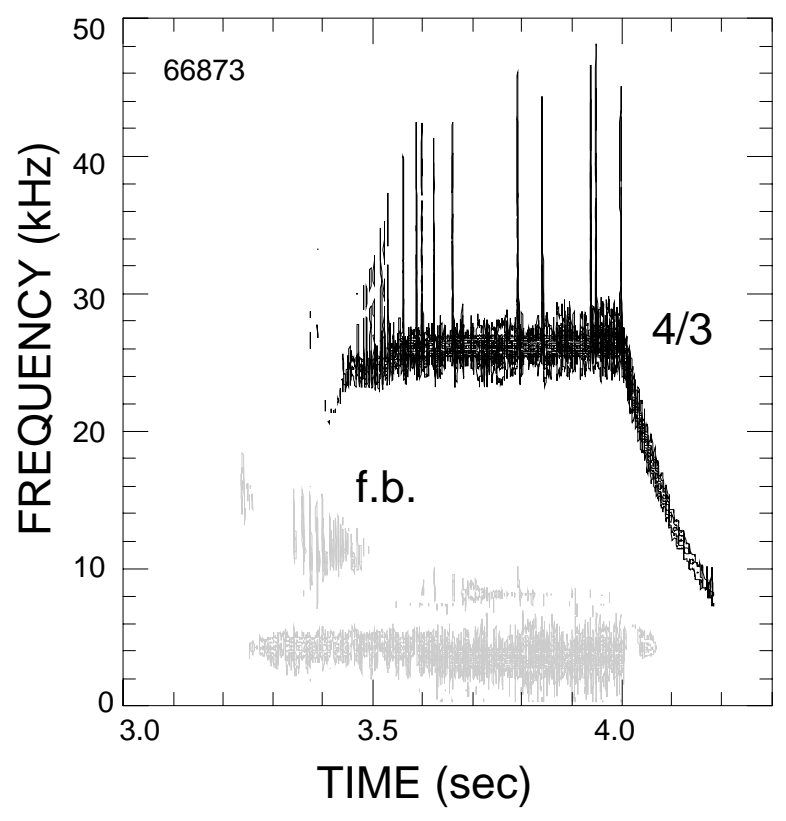

Fig. 4 Spectrogram of Mirnov coil data for shot 66873 showing evolution of 4/3 NTM and presence of bursting, fishbone-like modes.

Most NTMs on TFTR have also a strong ideal component present, dominantly, as a kink-like distortion of the plasma at the $q=1$ surface. This is generally described as a coupling to a mode with $m=n$, where $n$ is the toroidal mode number of the tearing mode. Thus, for the 4/3 NTM shown in the previous figures, there is also a 3/3 component.

This can be seen in Fig. 5 where profiles separated by half a period are shown. The perturbation caused by a $4 / 3$ mode should have been localized near the 4/3 surface. Instead, a perturbation can be seen which encompasses the entire core plasma. On the bad curvature 
side, the $3 / 3$ and of the $4 / 3$ are phase locked such that the displacements add, producing a nearly constant displacement from the $q=4 / 3$ surface to the magnetic axis. This mode structure is reminiscent of the structure of the 1/1 mode and may substantially reduce the stabilizing contribution from the field line bending energy.

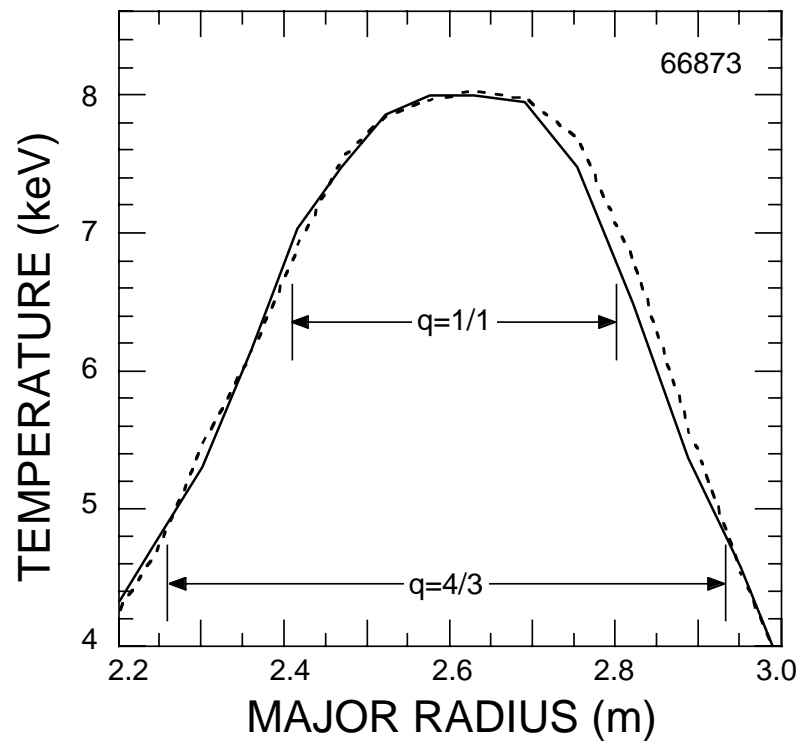

Fig. 5 Profiles of the electron temperature separated by one half period of the $4 / 3$ mode oscillation, illustrating the radial structure of the $4 / 3$ mode.

In Fig. 6 is shown a comparison of the simulation of the island width evolution to the measured magnetic fluctuation amplitude, where the simulated magnetic fluctuation level at the wall is inferred from the island width. The comparison of magnetic fluctuation levels, rather than the simulated vs. "measured" island widths accentuates disagreement, as the island width scales as the square root of the magnetic fluctuation level. The growth and decay of the island width is reasonably well matched. However the decay of the island width following the end of NBI heating is not well fit for many of the other $4 / 3$ modes. The multiplier on the neoclassical term, $\mathrm{k}_{\mathrm{nc}}$, is $\approx$ 1.25 compared to a range from $0.9-1.3$ for the eleven $4 / 3$ modes in this sequence.

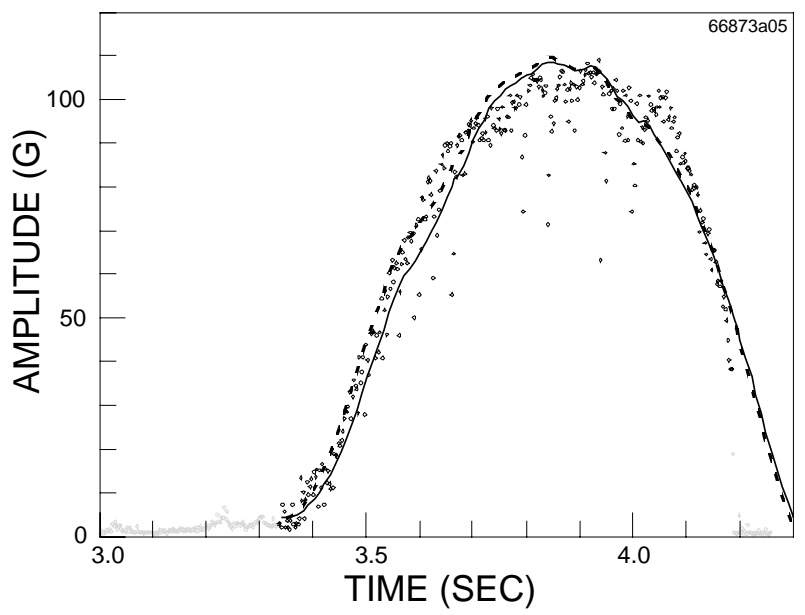

Fig. 6 Comparison of measured (dots) magnetic fluctuation amplitude for the shot shown in Figs. 3-4. The solid and dashed lines are simulations as described in the text.

The initial island growth in this case is approximately modeled by choosing a multiplier, $\mathrm{k}_{\mathrm{d}}$, on the critical island width, $\mathrm{w}_{\text {crit }}{ }^{2}$ $=\mathrm{k}_{\mathrm{d}} \mathrm{w}_{\mathrm{d}}^{2}$, to give a critical island width of about $3.5 \%$. Using the collisionless model for parallel transport, this multiplier is about 1.6. This critical island width results in a "trigger" island width of less than approximately $1.4 \%$. Two simulations are shown. The dashed line is a simulation using the local thermal $\beta_{\mathrm{pol}}$ to estimate the local bootstrap current. The solid 
line uses the TRANSP calculation of the local bootstrap current.

The mode terminates at the sawtooth crash, visible at $4.2 \mathrm{~s}$ in Fig. 3. The simulation shows virtually no effect of the sawtooth crash on the mode evolution, as would be expected since the neoclassical drive is already substantially reduced by this point and the effect of the current profile modification within the $q=1$ surface on mode stability is very small. Again, this suggests some other physics is playing a large role in the mode dynamics.

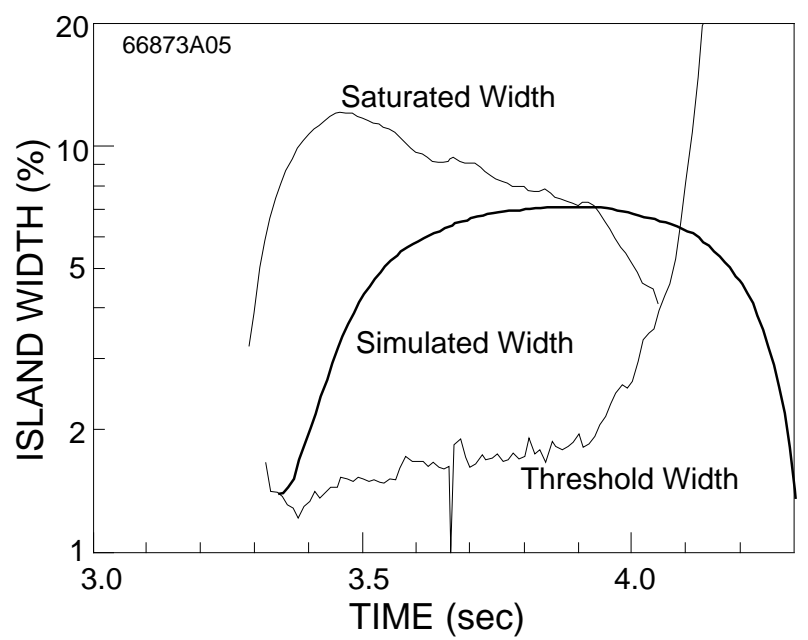

Fig. 7 Calculated threshold and saturated widths for the $4 / 3$ modes using the coefficients found by fitting magnetic fluctuation amplitude evolution in Fig. 6. Also shown is the simulated island width evolution from the simulation in Fig. 6.

The mode onset occurs shortly after it becomes metastable. In Fig. 7 are shown the time dependence of the saturated and threshold island sizes. The onset of the instability window is quite fast and the mode appears shortly thereafter.

Using the coefficients found by fitting the evolution of the $4 / 3$ mode, we can calculate the threshold and saturated island size for other modes. We find that the $2 / 1,3 / 2$ and $5 / 4$ modes all have predicted threshold island widths comparable to that of the $4 / 3$ mode, $\approx$ $1.2-1.5 \%$. The maximum predicted saturated widths are slightly greater than $10 \%$ for the $3 / 2$ and 5/4 modes, and $20 \%$ for the $2 / 1$ mode.

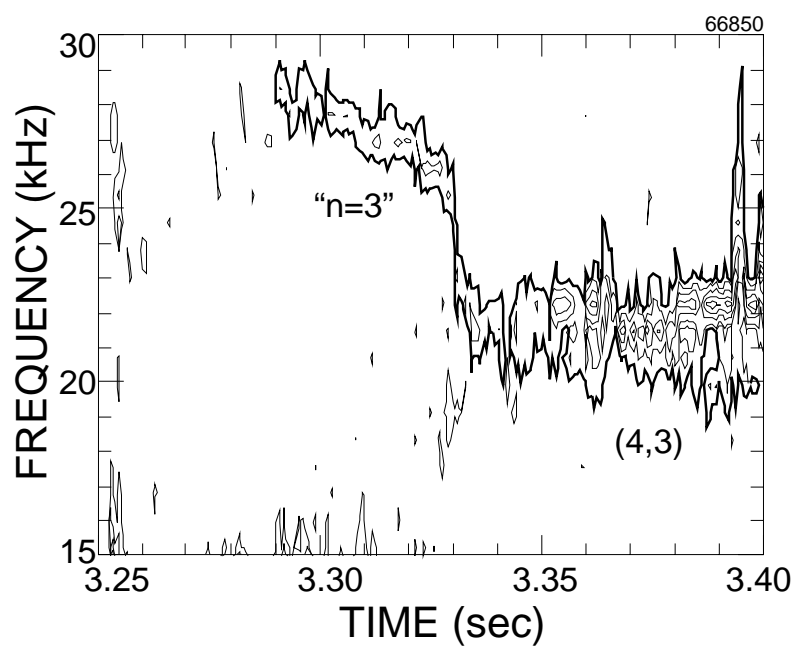

Fig. 8 Spectrogram of Mirnov coil data for shot 66850 showing onset evolution of 4/3 NTM.

In a second example the mode growth is very slow for a period of $>50 \mathrm{~ms}$ following first detection of the mode. In Fig. 8 is shown a spectrogram showing the onset of the mode, and in Fig. 9 is the simulated initial growth compared to that observed in the experiment. This long period of slow growth can be fit with this model, but the fit puts a tight constraint on the trigger island width. For the 
best fit $\mathrm{k}_{\mathrm{nc}}$ and $\mathrm{k}_{\mathrm{d}}$, the threshold width is $\approx$ $1.028 \pm 0.03 \%$.

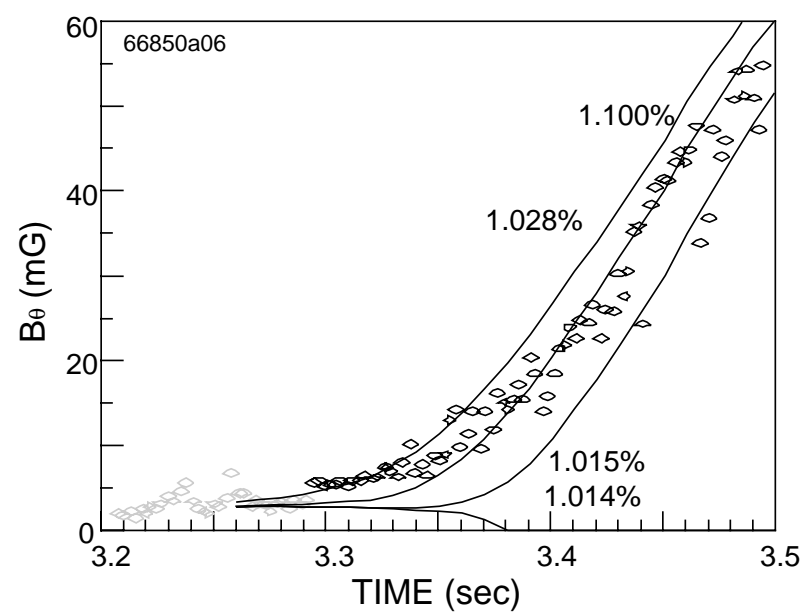

Figure 9 Comparison of measured (dots) and simulated (solid line) magnetic fluctuation amplitude showing quasi-saturated magnetic fluctuation amplitude after mode onset.

While it is not impossible that some NTMs are triggered at near the marginal stability point; the narrowness of this point contrasted with the relatively common occurrence of the slow initial growth suggest that this is not the explanation.

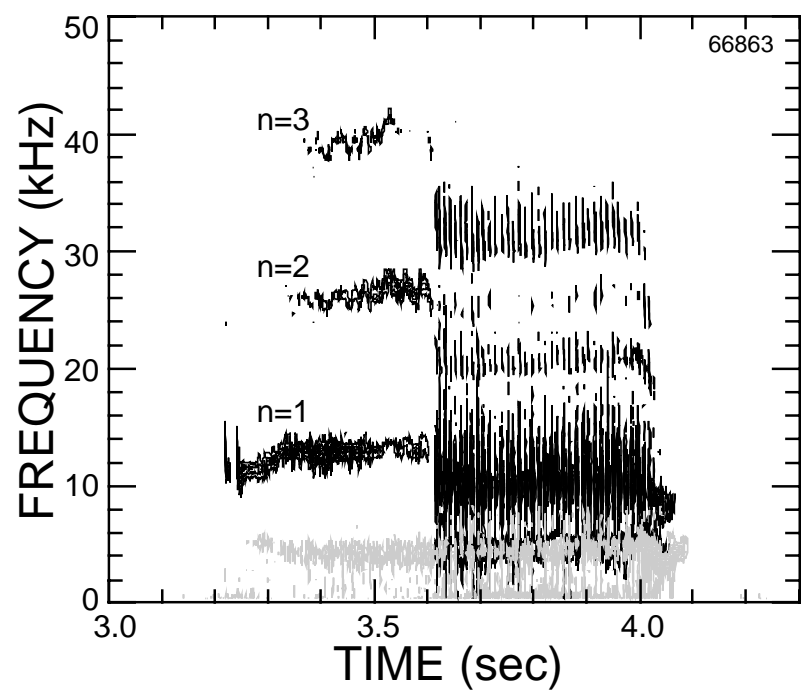

Fig. 10 Spectrogram of magnetic fluctuations.
Further, the frequency evolution shown in the spectrogram suggests that in the initial phase the character of the mode is in some way different from the latter "NTM period". The mode enters the "linear" growth phase at approximately the time of the frequency jump, consistent with the mode character evolving towards being NTM-like.

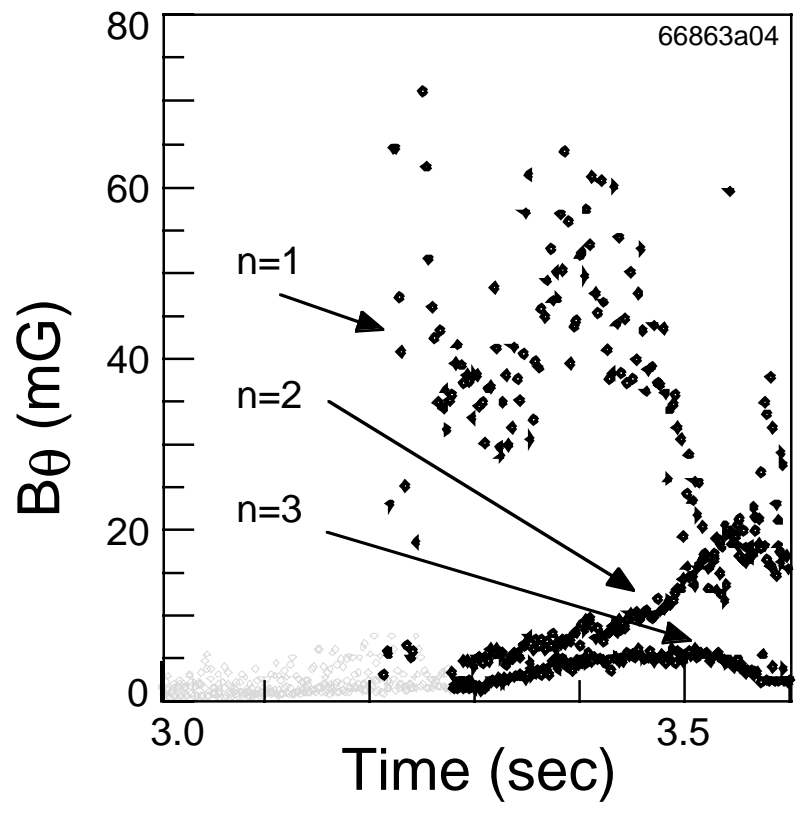

Fig. 11 Amplitude evolution of the $n=1, n=2$ and $n=3$ modes shown in Figure 7.

If the mode at onset is indeed a different type of mode, e.g., an ideal kink-like mode, then it might be expected that sometimes it doesn't drive a reconnection and form an island above the threshold size. In fact, such behavior is sometimes seen, as in the example shown in Figs. 10-12. In the first figure (Fig. 10) is shown a spectrogram of the magnetic fluctuations where the $n=1, n=2$ and $n=3$ modes are indicated. The toroidal mode 
numbers quoted here are the nominal toroidal mode numbers for the dominant component seen with the Mirnov coils. As will be shown below, it is believed that the modes have a complicated structure.

These modes are clearly not NTMs as they remain at very low amplitude before disappearing, as indicated in Fig. 10. These amplitudes would correspond to island widths of $\approx 1.3 \%$ for the $3 / 2$ and $\approx 1.2 \%$ for the $4 / 3$ mode. These inferred widths are comparable to the threshold island widths inferred above for $3 / 2$ or $4 / 3$ NTMs.

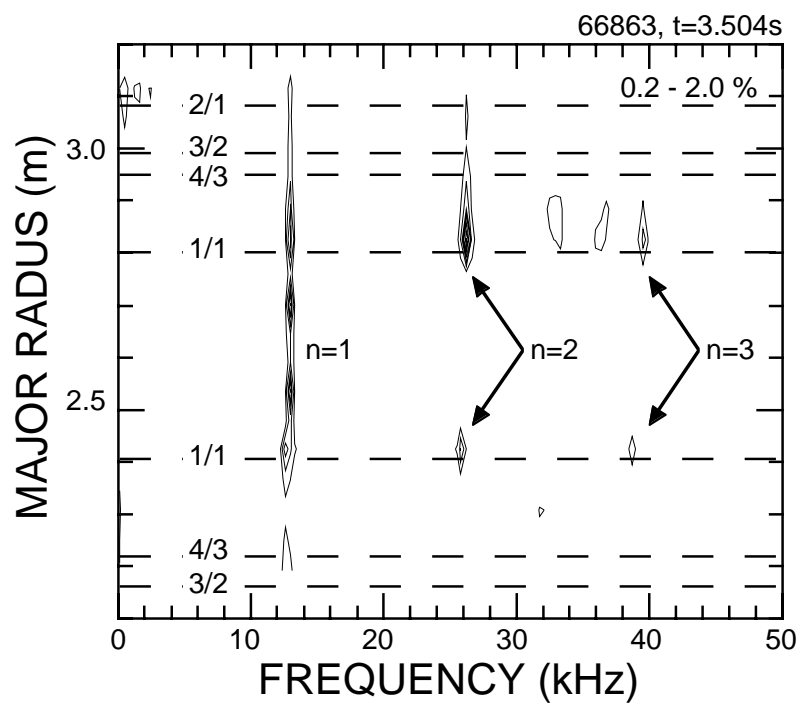

Fig. 12. Electron temperature fluctuations at 3.504s vs. frequency and major radius. Approximate locations of rational surfaces are marked.

In Fig. 12 the location of these modes is seen to be between the $\mathrm{q}=1$ and $\mathrm{q}=2$ surfaces. In this figure the electron temperature fluctuations are shown vs. major radius and frequency in a time window around $3.5 \mathrm{~s}$. The coherent fluctuations corresponding to the n=1, 2 and 3 modes shown in Fig. 10 are indicated. Additional coherent modes are also seen which are not detected by the Mirnov coils, suggesting that these other modes are more strongly localized to the core region. The $n=1-3$ modes are clearly associated with the $\mathrm{q}=1$ surface, but extend as far out as the $4 / 3,3 / 2$ or $2 / 1$ surfaces.

A striking feature of these modes is that the frequency peak on the inboard side appears to be at a lower frequency than on the outboard side (Figs. 12 and 13). The implication is that the width of these peaks in frequency reflects the presence of several "modes" at slightly different frequencies, with some being anti-ballooning, others having a weak ballooning character.

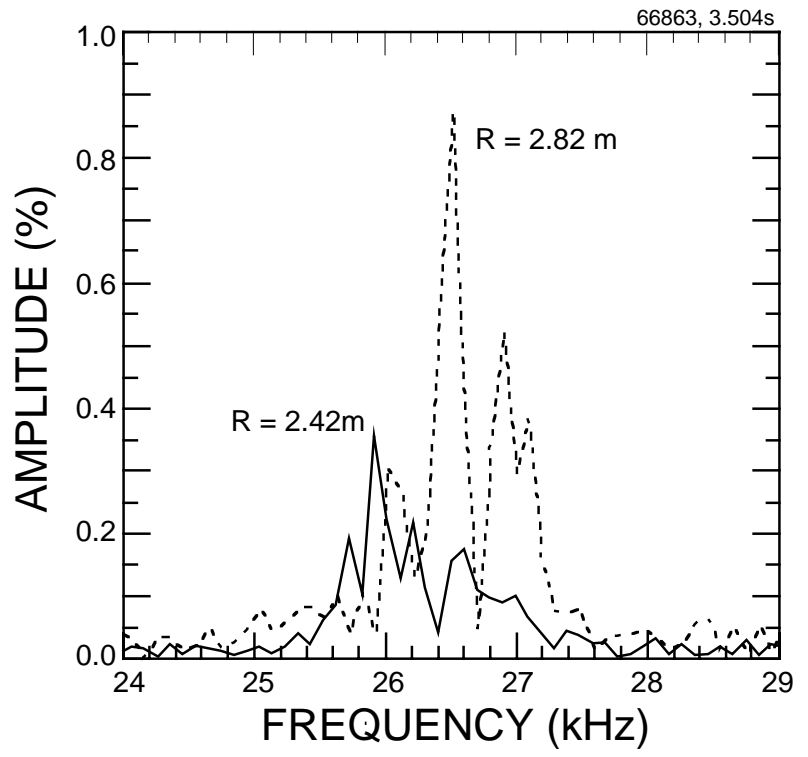

Fig. 13 Spectra of Te fluctuations from in and outboard sides showing " $\mathrm{n}=2$ mode".

In the next example we examine the behavior of multiple NTMs. This example has 
a nearly spontaneous $3 / 2$ mode and $4 / 3$ and $5 / 4$ modes. The $3 / 2$ is nearly spontaneous, as the trigger for the mode is a relatively mild, offaxis, reconnection event.

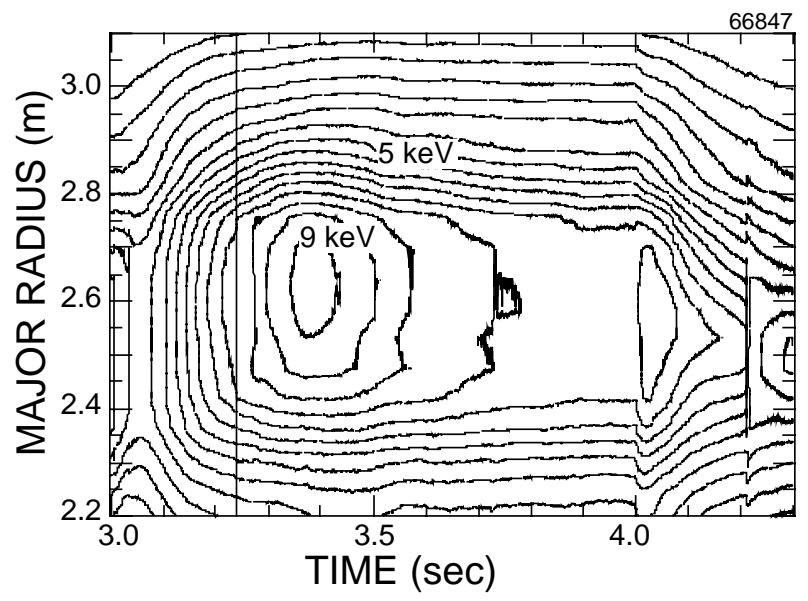

Fig. 14 Contours of electron temperature for shot $66847(3 / 2,4 / 3,5 / 4)$.

From the electron temperature profile evolution shown in Fig. 14, it can be seen again that there is very little electron temperature perturbation at the radius of the $3 / 2$ surface. Secondly, from the magnetic spectrogram shown in Fig. 15 it is seen that the off-axis sawtooth event had an undetectably weak precursor, at least as measured with the Mirnov coil. Thus, although the onset of this mode, and others, is clearly correlated with the off-axis reconnection, evidence for a direct, causal linkage is missing.

In Fig. 16 the simulated evolution of the $4 / 3$ edge magnetic fluctuation is compared to the measured fluctuation amplitude. The neoclassical multiplier is 1.17 compared to the range from 0.8 to 1.2 for the other seven cases of $3 / 2$ modes in this sequence. The initial growth was again slow and it was necessary to use a multiplier on the critical island width of $\approx 1.3$, in rough agreement with the factor of 1.6 needed for the 4/3 mode in Fig. 5.

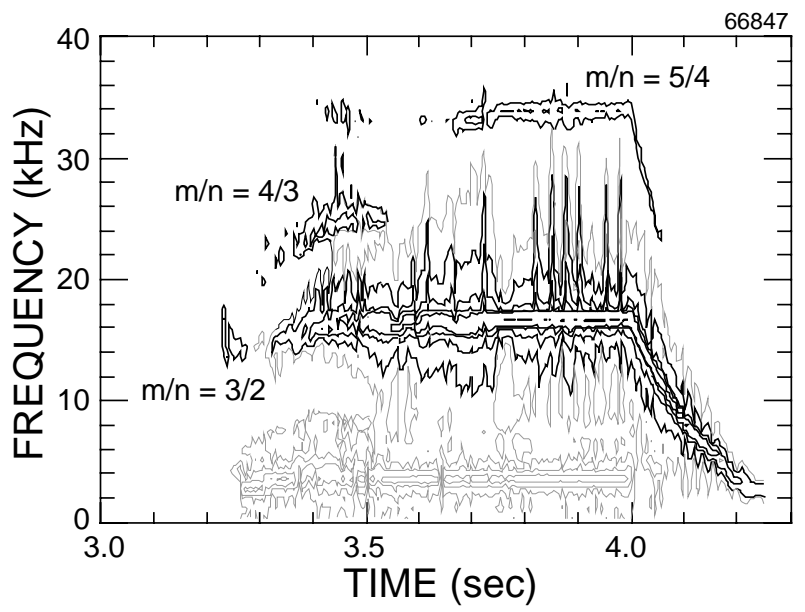

Fig. 15 Spectrogram of Mirnov coil data for shot 66847 showing onset of $3 / 2$ and 4/3 NTMs.

In this example there was also a $4 / 3$ mode triggered about the same time as the $3 / 2$ mode and a 5/4 mode which onset later. The 5/4 mode is well fit with a neoclassical multiplier of 1.0 , in quite reasonable agreement with the multiplier needed to fit the other NTMs in this sequence. However, the neoclassical multiplier needed to fit the $4 / 3$ mode evolution was only $\mathrm{k}_{\mathrm{nc}} \approx 0.41$. Even though the inferred $4 / 3$ island width reached $\approx 3 \%$, presumably well above the threshold island size, the mode suddenly disappeared after 3.5s. (The threshold island size inferred for the previous examples ranged from $\approx 1-1.5 \%$.) 
Similar behavior was also seen in the other three cases where $4 / 3$ and $3 / 2$ modes coexisted. This was true whether the $4 / 3$ mode onset before or during the $3 / 2$ mode activity. Note, however, that the $3 / 2$ and 5/4 modes are both well fit by the theory.
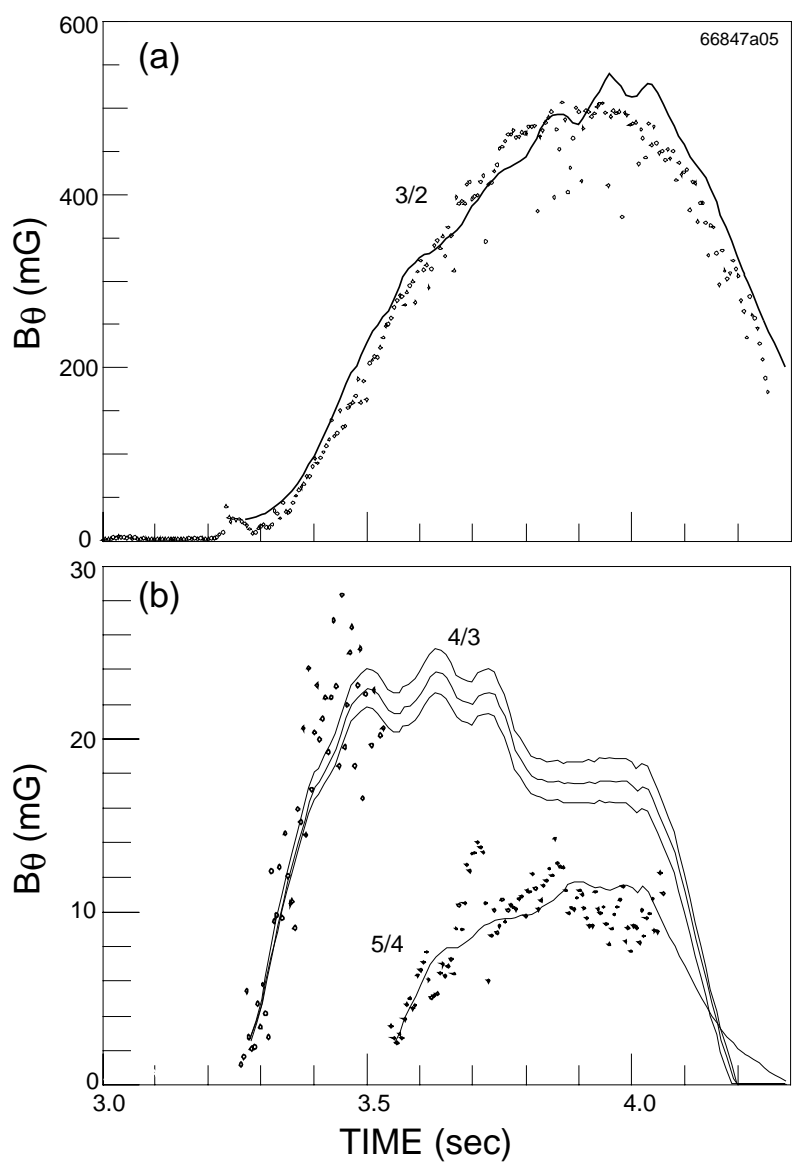

Fig. 16 Simulations of the amplitude evolution of the a) $3 / 2$, and b) $4 / 3$ and $5 / 4$ modes.

In the final example, we will examine one other situation in which the NTM theory does a poor job of predicting the $4 / 3$ mode evolution. Sawtooth crashes during saturated NTM activity result in a nearly instantaneous decrease in the inferred island size; a result not predicted by the theory. In three of the eleven shots in which 4/3 NTMs were the dominant mode, a sawtooth crash occurred during the quasi-saturated phase.

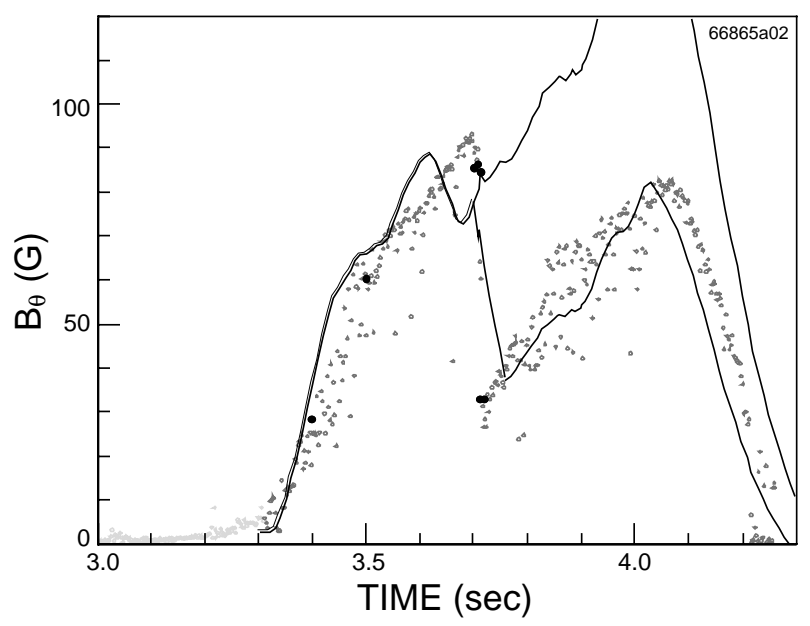

Fig. 17 Comparison of measured (dots) and simulated (solid line) magnetic fluctuation amplitude for a $4 / 3$ mode through a sawtooth crash. The simulation was done in three steps with the neoclassical term turned off following the sawtooth crash. (shot 66865)

In Fig. 17 two simulations of the $4 / 3$ mode evolution are compared to the measured mode amplitude. The first simulation keeps all adjustable parameters constant. There is almost no predicted effect of the sawtooth on the mode evolution. This is expected as the local density and temperature gradients at the $4 / 3$ surface, as well as q, are not expected to be affected by the sawtooth crash. However during the sawtooth crash, the measured magnetic fluctuation amplitude drops by nearly $50 \%$, implying a $25 \%$ reduction in island size, over a period shorter than $\approx 0.1$ $\mathrm{ms}$. This inferred rapid drop in island width is also supported by the GPC electron 
temperature profile measurements which also show a reduction in the local mode amplitude.

For the second simulation, the neoclassical drive term was set to zero for $57 \mathrm{~ms}$ after the sawtooth crash. The resistive time scale for island decay brought the predicted fluctuation level down to the measured level at that time; still a much slower drop than experimentally measured, implying the reduction in mode amplitude involves additional physics.

In this first set of data we have illustrated many of the observations which challenge the standard paradigm for tearing modes at high $\beta$. We will use a second data set to make two final points. The first is that the sawtooth stabilization threshold scales with the tearing mode onset threshold over the full range of parameters for TFTR. Secondly, in this set of data the range of necessary $\mathrm{k}_{\mathrm{nc}}$ 's to fit the NTM evolution is roughly half that used to fit the data presented above.

This second data set is from a sequence of approximately 15 discharges, of which 9 had the $\beta$ above the sawtooth stabilization threshold. The toroidal field was nominally 5.6T, neutral beam heating power was up to 40 $\mathrm{MW}, \mathrm{q}(\mathrm{a}) \approx 4$, and the plasma current was $\approx$ 2.6 MA. Of the sawtooth-stabilized discharges, four had $4 / 3$ modes, one had a $3 / 2$ mode and four had just fishbone-like modes.
(The 3/2 mode in this case was triggered by a minor disruption.)

The sawtooth stabilization threshold and the NTM threshold can be seen in Fig. 18. The threshold has dropped from $\beta_{\mathrm{pol}} \approx 1$ to $\beta_{\mathrm{pol}} \approx$ 0.5 as the $\mathrm{q}(\mathrm{a})$ has dropped from 5 to 4 . In both data sets, large sawteeth were present in the plasmas with $\beta$ just below the threshold. And in plasmas with $\beta$ above the threshold, large fishbone modes were also present.

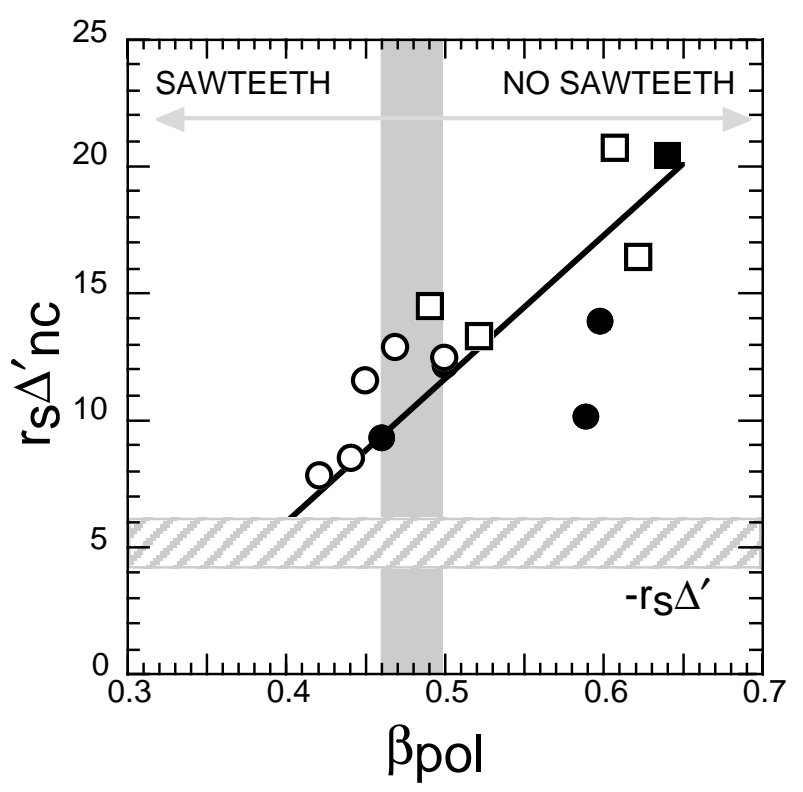

Fig. 18 Peak value of neoclassical drive term for the $4 / 3$ mode for 14 plasmas vs. poloidal beta. The hashed band are the complemetary range the approximate range of $\mathrm{D} \phi$. The open circles are sawtoothing plasmas, the open squares are fishboning plasmas. The solid circles are $4 / 3$ modes and the solid square is a $3 / 2$ mode.

The mode evolution is reasonably well fit with the NTM model (Fig. 19), but in these cases the typical multiplier on the $\Delta_{\mathrm{nc}}$ term is roughly a factor of 2 smaller than was the case at higher q(a). Also, to match the initial mode 
evolution, the multiplier on the $\mathrm{w}_{\text {crit }}$ is only 0.2 as compared with multipliers somewhat greater than unity in the previous data set. This was true for the four $4 / 3$ cases as well as the $3 / 2$ mode in this data set.

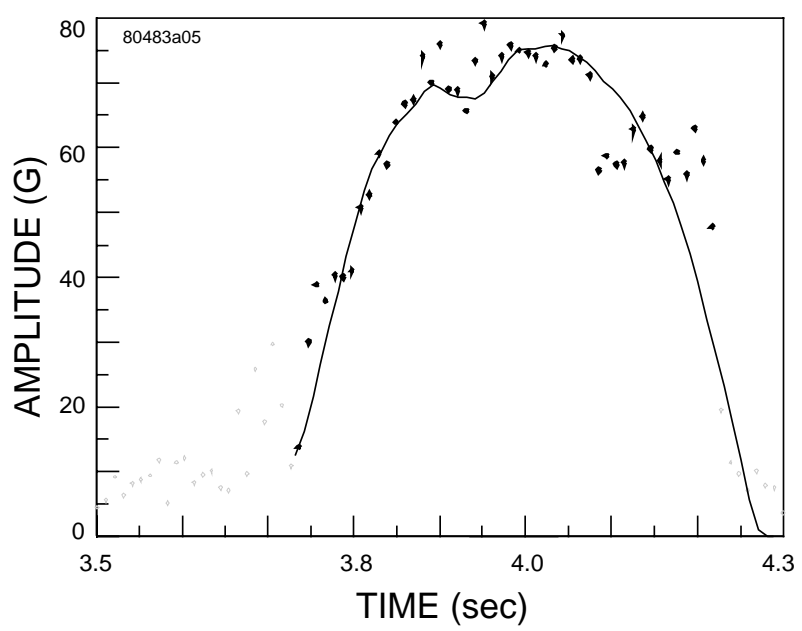

Fig. 19. Fit to mode amplitude evolution for a 2.6 MA, 5.6 $\mathrm{T}$ supershot plasma. The $\mathrm{k}_{\mathrm{nc}}$ used here is 0.42 .

\section{Discussion}

The neoclassical theory of tearing modes has been shown to reasonably well model the amplitude evolution of tearing modes under a wide range of conditions. It is incomplete in several aspects, however:

1) NTMs can clearly occur spontaneously - no extrinsic MHD is required.

2) Initial growth is not well fit by NTM theory; the very slow onset is difficult to reconcile with the hard onset predicted by the theory.

3 ) NTMs have a chirping character, implying some strong interaction with fast ions.
4) Constants in the Rutherford equation change for different conditions, machines.

5) NTM evolution is not well fit through the sawtooth crash.

6 ) Multiple mode evolution is only sometimes fit by theory.

The first point is one of the most serious. The fault with the original construction of NTM theory was that a much larger number of modes were predicted to be unstable than were observed. This prediction was robust in that the plasmas were well above the marginal $\beta$, and the predicted saturated amplitude of the modes was large. The theory could only be reconciled with experimental observations when viable threshold models were introduced. The observation of spontaneous NTMs invalidates these threshold models and again it is necessary for a major modification of the theory.

We propose here that the NTMs in TFTR are initially unstable ideal modes which can saturate at a fairly low level. If these modes reach sufficient amplitude, they force a reconnection and the mode takes on some of the character of an NTM. This assumption has a broad impact on NTM theory. Near the ideal marginal stability point, the $\Delta^{\prime}$ becomes large and positive, possibly negating the need for trigger islands. Without an understanding of 
the nature of this failure of ideal theory, it is difficult to predict stability conditions for next step machines.

The hypothesis that ideal instability plays a big role is also supported by the data from which point 2) was generated. The slow initial growth of tearing modes as illustrated in Figs. 8 and 9 is nearly impossible to reconcile with NTM theory as generally implemented, whereas some nonlinear ideal theories allow for saturation at a low amplitude.

Further direct evidence for ideal instability was presented in Figs. 10-13 where low amplitude, saturated $n=2$ and $n=3$ modes were clearly shown. These modes apparently did not reach sufficient amplitude to evolve into NTMs. The initial instability was driven by other processes, and was probably ideal.

The chirping character of the modes was explicitly pointed out in Fig. 4, but was present for all of the cases of TMs shown here, and more generally is a generic trait of these modes on TFTR. A chirping character is often assumed to indicate some fast ion drive for the modes. In this case, it is difficult to see how fast ions could be strongly resonant with these modes at such low frequency. However, TMs and the fishbone-like modes clearly enhanced the measured fast ion losses, and at each chirp there was a measurable drop in the neutron rate. The physical mechanism responsible for the chirping of the modes remains obscure, but this evidence suggests fast ions may be playing a role.

The fourth point would follow from the implications of the first three. Even with the more accurate estimate of the bootstrap current drive using the TRANSP code, the constants in the NTM theory needed adjustment over a range of two within the TFTR database. If an ideal pressure or fast ion drive is playing a role in the onset of the "NTMs", then it is not unreasonable to assume that it plays a role in the saturation amplitude as well. As the variation (scaling) of the pressure drive need not follow exactly the scaling of the bootstrap current drive, it is not so surprising that the constants in NTM theory must be adjusted for different parameter ranges.

The fifth point may fit with the model proposed above only if the damping rate for the ideal component of the mode is much faster than the resistive relaxation time. As shown in Fig. 17, the classical damping rate for the tearing mode is too slow to reduce the island size at the rate observed experimentally. The model is that the "NTM", as measured with the Mirnov coils, consists of the tearing mode contribution and a contribution from an ideal component. At the sawtooth crash it is possible that the ideal contribution, driven by the pressure gradient or fast ions inside the 
tearing mode rational surface, is greatly reduced in amplitude.

The last point is not clearly connected with the first four. A model has been proposed to explain a similar observation on ASDEX-U that $4 / 3$ and $3 / 2$ NTMs would not co-exist [23]. This model was based on cylindrical modeling of the tearing mode eigenfunctions, and it is not clear that those assumptions are valid for tearing modes with a strong ideal component, as are seen here.

\section{Summary of results}

We have presented clear experimental evidence that NTMs can occur without the traditional trigger sources (sawtooth crashes, fishbones or large ELMs). This observation is difficult to reconcile with the present standard model of tearing modes. We have also shown that under four other conditions, the observations are not consistent with the standard NTM theory.

The first is the case of multiple helicity NTMs where the 4/3 NTM is strongly damped in the presence of a $3 / 2$ NTM. This may eventually be understood in terms of a model of the form suggested by $\mathrm{Yu}$, et al. [23]. However, in the same shot the 5/4 mode was seemingly unaffected by the presence of the $3 / 2$ mode.
The second is the effect of a sawtooth crash on an NTM. In a very short time, less than a millisecond, the perceived NTM amplitude drops by a factor of $30 \%$. While the theories governing healing of magnetic islands are less well developed than those for the growth, this rapid healing is difficult to reconcile with what is expected for resistive mode behavior.

The third example is the fairly common (on TFTR) observation that many NTMs onset with a relatively long period of very slow island growth. This can conceivably be modeled with the resistive threshold island model, but the frequency of occurrence, given the tight constraints on the size of the trigger island make this explanation unlikely. In the final example, low amplitude modes are observed to persist for hundreds of milliseconds in a quasi-saturated state.

All of these observations are possibly consistent with a pernicious presence of an ideal component to the NTM. This would help to explain the frequency chirping typically observed for NTMs. The implications of this observation are that suppressing sawteeth, ELMs or other common extrinsic MHD triggers for NTMs will not guarantee stability.

\section{Acknowledgements}


The author is grateful to the TFTR team for their tireless support of the experiments from which this data was derived. This work was supported by U.S. DOE Contract DEAC02-76CH0307 


\section{Bibliography}

[1] S. V. Mirnov, I. G. Semenov, Sov. Journal of Atomic Energy 30, 22 (1971) or Sov. Phys. JETP 33, 1134 (1971).

[2] H.P. Furth, J. Killeen, and M.N. Rosenbluth, Phys. Fluids 6, (1963) 459.

[3] P. H. Rutherford, Phys. Fluids 16, 1903, (1973).

[4] R. B. White, D. A. Monticello, M. N. Rosenbluth, and B. V. Waddell,., Phys. Fluids 20, 800 (1977).

[5] D. Meade and the TFTR Group, in Proceedings of the International Conference on Plasma Physics and Controlled Nuclear Fusion, Washingon, DC, 1990 (International Atomic Energy Agency, Vienna, 1991), Vol. I, pp. 9-14).

[6] J.D. Callen, W.X. Qu, K.D. Siebert, B.A. Carreras, K.C. Sharing, and D.A. Spong in Plasma Physics and Controlled Nuclear Fusion Research 1986, Kyoto (International Atomic Energy Agency, Vienna, 1987), Vol. 2, p. 157; also see National Technical Information Service Document No. DE_-86008946/LL (W. X. Qu and J. D. Callen, University of Wisconsin Report No. UWPR-85-5, 1985). Copies may be ordered from National Technical Information Service, Springfield, Virginia 22161.

[7] R. Carrera, R. D. Halzeltine, and M. Kotschenreuther, Phys. Fluids 29, 899 (1986).

[8] R. Fitzpatrick, Phys. Plasmas 2, 825 (1995). Callen, Varrena?

[9] A. I. Smolyakov, A. Hirose, E. Lazzaro, G. B. Re, J. D. Callen, Phys. Plasmas 2, 1581 (1995).

[10] H. R. Wilson, J. W. Conner, R. J. Hastie, and C. C. Hegna, Phys. of Plasmas 3, 248 (1996).

[11] F. Waelbroeck and R. Fitzpatrick, Phys. Rev. Lett. 78, 1703 (1997).

[12] Z. Chang, J. D. Callen, E. D. Fredrickson, R. V. Budny, C. C. Hegna, K. M.

McGuire, M. C. Zarnstorff, and the TFTR Group, Phys. Rev. Lett. 74, 4663 (1995).

[13] R.J. LaHaye, L.L. Lao, E.J. Strait, T.S. Taylor, Nucl. Fusion 37, 397 (1997).

[14] D. A. Gates, B. Lloyd, A. W. Morris, G. McArdle, M. R. O’Brien, M. Valovic, C. D. Warrick, H. R. WIlson, COMPASS-D Team, ECRH Team, Nucl. Fusion 37, 1593 (1997).

[15] R. Kaita, R.B. White, A.W. Morris, et al., Phys. Fluids B 2, 1584 (1990).

[16] R.V. Budny, Nucl. Fusion 34, 1247 (1994), and references therein.

[17] A. H. Glasser, J. M. Greene, and J. L. Johnson, Phys. Fluids 18, 875 (1975).

[18] E. Fredrickson, M. Bell, R.V. Budny, and E. Synakowski, Phys. of Plasmas 7, (2000), p. 4112.

[19] N.N. Gorelenkov, R.V. Budny, Z. Chang, M.V. Gorelenkova, and L.E. Zakharov, Phys. Plasmas 3, 3379 (1996).

[20] T. A. Gianakon, C. C. Hegna, J. D. Callen, Phys. Plasmas 3, 4637 (1996). 
[21] Z. Chang, E. D. Fredrickson, S. H. Batha, M. G. Bell, R. V. Budny, F. M. Levinton, K. M. McGuire, G. Taylor, M. C. Zarnstorff and the TFTR Group, Phys. of Plasmas 5, 1076 (1998).

[22] S. Sesnic, S. Gunter, A. Gude, M. Maraschek, and the ASDEX Upgrade Team, Phys. of Plasmas 7, 935 (2000).

[23] H. Zohm, G. Gantenbein, A. Gude, S. Gunter, F. Leuterer, M. Maraschek, J.P. Meskat, W. Suttrop, Q. Yu, ASDEX Upgrade Team, ECRH Group (AUG), Nucl. Fusion 41, 197 (2001). 


\section{External Distribution}

Plasma Research Laboratory, Australian National University, Australia

Professor I.R. J ones, Flinders University, Australia

Professor J oão Canalle, Instituto de Fisica DEQ/IF - UERJ , Brazil

Mr. Gerson O. Ludwig, Instituto Nacional de Pesquisas, Brazil

Dr. P.H. Sakanaka, Instituto Fisica, Brazil

The Librarian, Culham Laboratory, England

Library, R61, Rutherford Appleton Laboratory, England

Mrs. S.A. Hutchinson, JET Library, England

Professor M.N. Bussac, Ecole Polytechnique, France

Librarian, Max-Planck-Institut für Plasmaphysik, Germany

J olan Moldvai, Reports Library, MTA KFKI-ATKI, Hungary

Dr. P. Kaw, Institute for Plasma Research, India

Ms. P.J . Pathak, Librarian, Insitute for Plasma Research, India

Ms. Clelia De Palo, Associazione EURATOM-ENEA, I taly

Dr. G. Grosso, Instituto di Fisica del Plasma, Italy

Librarian, Naka Fusion Research Establishment, J AERI, J apan

Library, Plasma Physics Laboratory, Kyoto University, J apan

Research Information Center, National Institute for Fusion Science, J apan

Dr. O. Mitarai, Kyushu Tokai University, J apan

Library, Academia Sinica, Institute of Plasma Physics, People's Republic of China

Shih-Tung Tsai, Institute of Physics, Chinese Academy of Sciences, People's Republic of China

Dr. S. Mirnov, TRINITI, Troitsk, Russian Federation, Russia

Dr. V.S. Strelkov, Kurchatov Institute, Russian Federation, Russia

Professor Peter Lukac, Katedra Fyziky Plazmy MFF UK, Mlynska dolina F-2, Komenskeho Univerzita, SK-842 15 Bratislava, Slovakia

Dr. G.S. Lee, Korea Basic Science Institute, South Korea

Mr. Dennis Bruggink, Fusion Library, University of Wisconsin, USA

Institute for Plasma Research, University of Maryland, USA

Librarian, Fusion Energy Division, Oak Ridge National Laboratory, USA

Librarian, Institute of Fusion Studies, University of Texas, USA

Librarian, Magnetic Fusion Program, Lawrence Livermore National Laboratory, USA

Library, General Atomics, USA

Plasma Physics Group, Fusion Energy Research Program, University of California at San Diego, USA

Plasma Physics Library, Columbia University, USA

Alkesh Punjabi, Center for Fusion Research and Training, Hampton University, USA

Dr. W.M. Stacey, Fusion Research Center, Georgia Institute of Technology, USA

Dr. J ohn Willis, U.S. Department of Energy, Office of Fusion Energy Sciences, USA

Mr. Paul H. Wright, Indianapolis, Indiana, USA 
The Princeton Plasma Physics Laboratory is operated by Princeton University under contract with the U.S. Department of Energy.

\author{
Information Services \\ Princeton Plasma Physics Laboratory \\ P.O. Box 451 \\ Princeton, NJ 08543
}

Phone: 609-243-2750

Fax: 609-243-2751

e-mail: pppl_info@pppl.gov

Internet Address: http://www.pppl.gov 